\title{
A Method for Determining the Screening Length of the Coulombic Scattering in Non-Degenerate and Degenerate Semiconductors
}

\author{
M. Rudan and G. Perroni \\ E. De Castro Advanced Research Center on Electronic Systems (ARCES), and \\ Department of Electronics, Computer Science and Systems (DEIS) \\ University of Bologna, Viale Risorgimento 2 \\ 40136 Bologna, Italy \\ Tel. +39-051-20-93016, Fax. -93779 \\ E-MAIL mrudan@deis.unibo.it, gperroni@deis.unibo.it
}

\begin{abstract}
A method for directly measuring the inverse screening length in semiconductors in terms of ionized-impurity concentration and lattice temperature is demonstrated, based on a first-principle calculation of the effect of ionized-impurity scattering. No fitting parameters are involved in the derivation, which covers a wide range of doping concentrations and intrinsically complies with the Mathiessen rule. A new class of integrals is introduced in the calculation. The results are compared with the standard derivation of the inverse screening length from the perturbative solution of the Poisson equation.
\end{abstract}

\section{Motivation}

Low-field measurements of carrier mobility, coupled with an analytical calculation of the momentum-relaxation time, provide a method for a direct experimental extraction of the dependence of the inverse screening length $q_{0}$ on the ionized-impurity concentration $N_{I}$ and lattice temperature $T_{L}$. This constitutes the basis for deriving a first-principle model of the effect of ionized-impurity scattering on mobility.

The concept of inverse screening length is adopted in a number of microscopic models for determining the effect of ionized-impurity scattering (e.g., Brooks-Herring, Takimoto, and Third-body exclusion models [1]). Such parameter, in turn, depends on the ionized-impurity concentration and lattice temperature through the charge density.

Some important issues that play a role in the effect of ionized-impurity scattering on the carrier mobility must be mentioned. The first issue is the questionable approximation of replacing the carrier concentration with the impurity concentration in the standard expressions involving $q_{0}$, which leads to an infinite collision cross-section at zero concentration. A second issue is the dependence of the ionized-impurity scattering on temperature, a third one is the different effectiveness of this type of scattering on the majority and minority carriers, which brings about the necessity of distinct mobility models as shown in [2], and a fourth one is the consistency of the result with the Mathiessen rule, which is often missing in models derived from first principles.

Here it is shown that combining the definition of the momentum-relaxation time typical of the hydrodynamic transport equations [3] with the concept of screening length 
provides a method for experimentally determining the dependence of the latter on the ionized-impurity concentration and temperature. While preliminary results have been shown in [4] for the non-degenerate case, here the theory is carried out in the full range of significant dopant concentrations $3 \times 10^{13}<N_{I}<10^{21} \mathrm{~cm}^{-3}$.

\section{Theory}

We begin with a set of measurements of the electron mobility $\mu_{n}$, carried out under a low-field regime and different temperatures on silicon slabs, each uniformly doped with a different impurity concentration. In such experimental condition, the two effects relevant for mobility are the phonon and the ionized-impurity scattering. In the standard models of semiconductor devices, the two contributions are combined into an expression for mobility based on the Mathiessen rule $\mu_{n}^{-1}=\mu_{P}^{-1}+\mu_{I}^{-1}$, where $\mu_{P}, \mu_{I}$ refer to the individual effects (the electron mobility $\mu_{n}$ is used here by way of example). In turn, the Mathiessen rule reflects the basic additivity property of incorrelated events' probability. The microscopic theory outlined below is carried out consistently with such property.

First, for each lattice temperature the ionized-impurity contribution is separated from the phonon contribution as

$\left[\mu_{I}\left(N_{I}\right)\right]_{\exp }=\frac{\mu_{n} \mu_{n}\left(N_{I} \rightarrow 0\right)}{\mu_{n}\left(N_{I} \rightarrow 0\right)-\mu_{n}}$

Then, the experimental result is equated to a theoretical expression $\left[\mu_{I}\left(N_{I}\right)\right]_{\text {theor }}$, which is derived by directly integrating the Boltzmann Transport Equation over the momentum space as a step of the formal procedure prescribed by the moments' method [3]. This yields the macroscopic relaxation-time tensor $\tau_{i v}$ related to the ionized-impurity scattering

$\frac{1}{\tau_{i v}}=\frac{\int_{v} u_{i}\left(f / \tau_{I}\right) \mathrm{d}^{3} k}{\int_{v} u_{i} f \mathrm{~d}^{3} k}, \quad \frac{1}{\tau_{I}}=\frac{8 \pi^{2} \omega_{v} m_{e} N_{I} Z_{c}^{2} e^{4}\left|\boldsymbol{k}-\boldsymbol{k}_{v}\right|}{\hbar^{3} \varepsilon_{\mathrm{sc}}^{2} q_{0}^{2}\left(q_{0}^{2}+4\left|\boldsymbol{k}-\boldsymbol{k}_{v}\right|^{2}\right)}$,

where $f$ is the distribution function, $u_{i}$ the $i$ th component of the group velocity, and $v$ the valley index within the Brillouin zone. In turn, $\tau_{I}$ is the microscopic relaxation time of the ionized-impurity scattering [5], with $\omega_{v}$ the dimensionless overlap factor of the valley, $m_{e}$ the average effective mass of the electrons, $Z_{c} e$ the charge of the Coulombic center, $\boldsymbol{k}_{v}$ the wave vector at the valley's bottom, and $\varepsilon_{\mathrm{sc}}$ the dielectric constant of the semiconductor.

In most operating conditions of the semiconductor devices the different scattering mechanisms are incorrelated. Labeling the individual effects as above and letting $\tau$ be the total microscopic scattering time, one finds $\tau^{-1}=\tau_{P}^{-1}+\tau_{I}^{-1}+\ldots$, whence the Mathiessen rule follows immediately. Note that definition (2) differs from that given, e.g., in $[1,6]$ and [7, Sect. 4.1] which for the $v$ th valley reads $\tau_{v}=\langle\tau E\rangle_{v} /\langle E\rangle_{v}$. The latter is found by calculating the average after multiplying both sides of the Boltzmann Transport Equation by $\tau$ and using the energy $E$ as integration variable. Although the calculations based on it may be less involved, such definition is eventually inconsistent with the use of the Mathiessen rule because

$\left\langle\left(\sum_{i} \tau_{i}^{-1}\right)^{-1}\right\rangle \neq\left(\sum_{i}\left\langle\tau_{i}^{-1}\right\rangle\right)^{-1}$. 
Taking a uniform material and applying a small, time-independent electric field $\mathcal{E}$, the electron-distribution function $f$ fulfills the steady-state, uniform Boltzmann equation, whose perturbative form leads to $f \simeq\left[1+\tau \boldsymbol{u} \bullet e \mathcal{E}\left(\mathrm{d} \log f^{\mathrm{eq}} / \mathrm{d} E\right)\right] f^{\mathrm{eq}}$. As no hypoth-

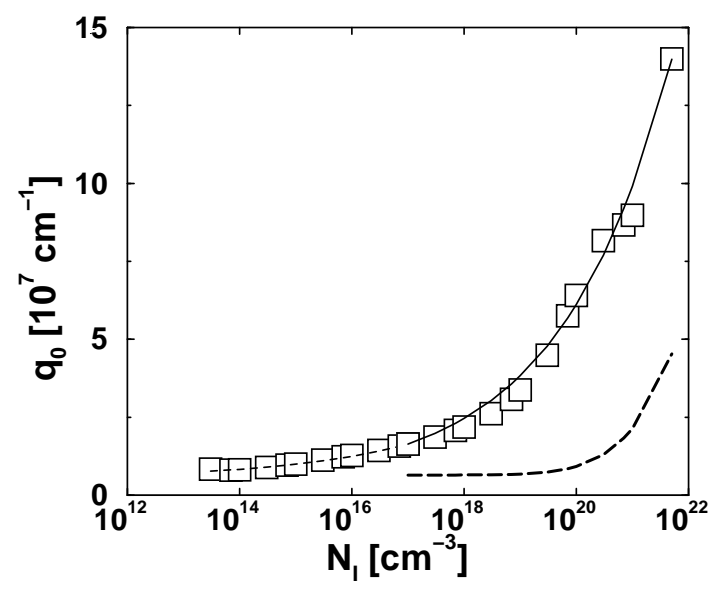

Figure 1: Inverse screening length $q_{0}$ vs the impurity concentration $N_{I}$ for electrons in $n$-doped silicon at $T_{L}=300 \mathrm{~K}$. Squares: experiment. The continuous line interpolates the experimental results in the degenerate region, the thin dashed line interpolates them in the non-degenerate region. Thick long-dashed line: perturbative solution of Poisson's equation obtained by extending the method of [1] to the degenerate region.

esis about the form of $f^{\text {eq }}$ is necessary for the derivation above, the result holds for both the degenerate and non-degenerate cases. Replacing $f$ into (2) leads, after a somewhat involved calculation and an average over the valleys, to

$\frac{N_{I}\left[\mu_{I}\left(N_{I}, T_{L}\right)\right]_{\exp }}{\exp \left(-\xi_{e}\right) \Phi_{1 / 2}\left(\xi_{e}\right)}=\frac{2 \theta \exp \left(-\lambda^{2} q_{0}^{2}\right)}{R_{0}\left(\lambda^{2} q_{0}^{2}, \xi_{e}\right)-R_{2}\left(\lambda^{2} q_{0}^{2}, \xi_{e}\right)}$,

with

$\lambda^{2}=\frac{\hbar^{2}}{8 m_{e} k_{B} T_{L}}, \quad \theta=\frac{3 \sqrt{2 m_{e}} \varepsilon_{\mathrm{sc}}^{2}\left(k_{B} T_{L}\right)^{3 / 2}}{\pi^{3 / 2} e^{3} Z_{c}^{2} m_{n}}$,

which provides the experimental determination of $q_{0}\left(N_{I}, T_{L}\right)$. In (4), $m_{n}$ is the transport effective mass of the electrons, $\xi_{e}$ the distance between the conduction-band edge and the Fermi level normalized to $k_{B} T_{L}$ (incomplete ionization is accounted for), $\Phi_{1 / 2}$ is the Fermi integral of order $1 / 2$, and $R_{0}, R_{2}$ are defined by

$R_{\alpha}\left(\rho_{0}, \xi_{e}\right) \doteq \int_{1}^{\infty} \frac{w^{-\alpha} \mathrm{d} w}{\exp \left(\rho_{0} w\right)+\exp \left(\rho_{0}+\xi_{e}\right)}$.

\section{Significance and Conclusion}

The method presented here allows for the direct measurement of a rather fundamental quantity, the inverse screening length $q_{0}$, as a function of $N_{I}$ and $T_{L}$. In the authors' 
knowledge, a method for measuring $q_{0}$ was not proposed before. Moreover, the theory depicted here provides the basis for modeling the contribution of the ionized-impurity scattering to the carrier mobility, in the full range of doping concentration and temperature, without the need of fitting parameters. In contrast with other methods based on first-principle calculations, the result is consistent with the Mathiessen rule, which in turn reflects the basic additivity property of incorrelated events' probability. Moreover, the direct extraction of $q_{0}$ intrinsically accounts for the differences due to the type of dopant, specifically, to the scattering effect of a given impurity concentration on the majority or minority carriers, which is known to be different [2]. The unphysical effect of a diverging collision cross-section for a vanishing concentration is absent in the procedure presented here, which inherently provides the screening length in terms of the ionized-impurity concentration.

The theory requires the definition of a new class of integrals (6). The problem of the practical calculation of (6) had to be tackled in the frame of this investigation because integrals of this type have not been studied before; however, further details are outside the scope of this work and will be given elsewhere.

Finally, the results indicate a more effective screening than that found from the standard method [1] of solving Poisson's equation by perturbation. This is visible in the figure, that shows $q_{0}\left(N_{I}\right)$ for electrons in $n$-doped silicon at $T_{L}=300 \mathrm{~K}$ (symbols: squares). A power law interpolating the experiments is also shown: the continuous (thin dashed) line refers to the $N_{I}>10^{17}\left(N_{I}<10^{17}\right)$ case. Finally, the lower, long-dashed line shows $q_{0}\left(N_{I}\right)$ as calculated from the perturbative solution of Poisson's equation. ${ }^{1}$

\section{References}

[1] B. K. Ridley, "Quantum Processes in Semiconductors", Oxford: Clarendon Press, 1993.

[2] D. B. M. Klaassen, Solid State Electronics, 35, 1992, p. 953 and p. 961.

[3] M. Rudan, M. Lorenzini, R. Brunetti, "Hydrodynamic Simulation of Semiconductor Devices", Electronic Materials, Vol. 4, Ch. 2, E. Schöll Ed., Chapman \& Hall, 1998.

[4] M. Rudan, G. Perroni, Proc. HCIS 13, Modena, July 2003.

[5] C. Jacoboni, L. Reggiani, Reviews of Modern Physics, 55, 1983, p. 645.

[6] P. Kiréev, "Le Physique des Semiconducteurs", Moscou: MIR, 1967.

[7] E. Conwell, "High Field Transport in Semiconductors", Accademic Press, 1967.

${ }^{1}$ Work partially supported by the EU-FP5 Project IST-2000-30033 "DEMAND". 